\title{
Comparative Ultrastructures of the Fertilized Egg Envelopes in Nothobranchius guentheri and Nothobranchius patrizii, Nothobranchiidae, Teleostei
}

\author{
Jung Kyon Kwon, Han Suk Jung ${ }^{1}$, Dong Heui Kim²,* \\ Department of Biomedical Laboratory Science, Kyungdong University, Goseong 24764, Korea \\ ${ }^{1}$ Department of Chiropractic, Hanseo University, Seosan 31962, Korea \\ ${ }^{2}$ Department of Environmental Medical Biology, Yonsei University Wonju College of Medicine, Wonju 26426, Korea
}

*Correspondence to:

$\mathrm{Kim} \mathrm{DH}$,

Tel: $+82-33-741-0332$

Fax: $+82-33-731-6953$

E-mail: fish7963@yonsei.ac.kr

Received August 3, 2015

Revised August 31, 2015

Accepted September 10, 2015
Nothobranchius guentheri and Nothobranchius patrizii have special life cycle to sustain the dry season. So, we investigated the fertilized eggs morphology, and compared ultrastructures of surface structures and the cross section of fertilized egg envelopes using light and electron microscopes to determine whether these fertilized eggs and egg envelopes show the species specificity or have special structure to sustain the dry season. These fertilized eggs were spherical, yellowish, demersal and adhesive, and had a one-sided large oil droplet. The whip-like structures, adhesive filament were distributed throughout egg envelope in both species. But, that of $N$. guentheri was covered with fibrous structures, and that of $N$. patrizii was smooth. The egg envelope consisted of two distinct layers: an outer, electron-dense layer containing adhesive filaments and an inner layer of 16 to 17 horizontal electron-dense lamellae alternating with 15 to 16 interlamellae of lower electron density in both species. The external shapes of fertilized egg and section of fertilized egg envelope were same, but ultrastructure of adhesive filaments on the outer surface was concluded to show species specificity. Our data indicate that the ultrastructural differences of adhesive filament and outer surface of fertilized egg envelope show species specificity although these species belong to same genus.

Key Words: Fertilized egg, Egg envelope, Nothobranchius guentheri, Nothobranchius patrizii, Ultrastructure

\section{INTRODUCTION}

Fish eggs are surrounded by an acellular structure, egg envelope. It plays a role in diffusive exchanges of gases such as carbon dioxide and oxygen, selective transport of materials into the egg, protections for physical damage (Donovan \& Hart, 1986) and from chemicals and pathogens, and polyspermy prevention (Harvey et al., 1983; Cameron \& Hunter, 1984). In teleost, the fine structure of the fertilized egg envelope differ according to the physiochemical characteristics of the water environment (Lönning, 1972). These ultrastructures have related with environmental factors (Stehr \& Hawkes,
1979), and type of spawning place (Ivankov \& Kurdyayeva, 1973). Also, the structure of the egg envelope differ according to species as well as by family (Deung et al., 1999; Kim et al., 2002). Even eggs of the same species have been reported to have different shapes depending on their geographical distribution (Brummett \& Dumont, 1981).

The Guentheri killifish (Nothobranchius guentheri) belong to Nothobranchiidae also known as the redtail notho. These species live in water holes, streams, and marshes in Africa (Huber, 1996). The food are mosquito larvae and plankton. During the dry season when the temporary pools of water the fish inhabit dry up and the adult fish perish, specially adapted

(a) This is an open-access article distributed under the terms of the Creative Commons Attribution Non-Commercial License (http://creativecommons.org/licenses/by-nc/4.0) which permits unrestricted noncommercial use, distribution, and reproduction in any medium, provided the original work is properly cited.

Copyrights @ 2015 by Korean Society of Microscopy 


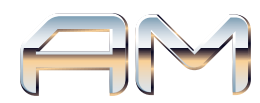

proteins in the egg envelope are triggered to retain moisture, thereby ensuring the next generation of fish last until the rains return and the fry can hatch (Bailey \& Sandford, 1998). Hatching enzyme is released to the egg envelope and allow the fish to hatch (Kawaguchi et al., 2010). Eggs have a dry incubation of between 8 and 12 weeks, but most hatch after 9 weeks. The blue notho (Nothobranchius patrizii) mostly found in Kenya and Somalia, and have same life cycle with readtail notho.

In such a reason, fertilized egg and egg envelopes of these species have to have a special structure to sustain the dry season. So, we investigated the fertilized egg morphology, and compared ultrastructures of surface structures and the cross section of fertilized egg envelopes of $N$. guentheri and $N$. patrizii with special life cycle using light and electron microscopes to determine whether these fertilized eggs and egg envelopes show the species specificity or have special structure to sustain the dry season.

\section{MATERIALS AND METHODS}

\section{Animals}

The fishes, $N$. guentheri and $N$. patrizii used in this study were purchased from Mr. Urai Wongwian (Thailand) in August, 2013. The tap water used for rearing was treated with AquaSafe (Tetra Co., Ltd., Germany) to remove chlorine, and its temperature and $\mathrm{pH}$ were maintained at $25^{\circ} \mathrm{C} \pm 1^{\circ} \mathrm{C}$ and $\mathrm{pH} 6.5 \pm 0.5$, respectively. Biological filtration was performed using a sponge filter (Brilliant sponge filter; Tetra Co., Ltd.), and excrement settled to the bottom of the water tank was eliminated by exchanging one-quarter of the water each week. An artificial light was illuminated for ten hours per day to simulate a daytime environment, and frozen bloodworms (Blood Worms; Hikari Sales USA Inc., USA) was provided as food two times per day at 9 a.m. and 5 p.m.

\section{Collection of Fertilized Eggs}

For the collection of fertilized eggs, each pair of fish was put into a glass water tank $(45 \times 45 \times 40 \mathrm{~cm})$, and a $250 \mathrm{~mL}$ beaker filled with peat moss as a spawning place was located on the corner in the glass tank. The fertilized egg was corrected from the peat moss after spawning. Fertilized eggs were measured for size $(\mathrm{n}=10)$ under light microscope and used as samples for morphological analysis.

\section{Electron Microscopy}

For transmission electron microscope (TEM) observation, fertilized eggs were fixed in $2.5 \%$ glutaraldehyde in $0.1 \mathrm{M}$ phosphate buffer ( $\mathrm{pH} 7.4$ ) for 2 hours at $4^{\circ} \mathrm{C}$. After prefixation, the specimens were washed twice in the same buffer solution and then postfixed in $1 \%$ osmium tetroxide solution in $0.1 \mathrm{M}$ phosphate buffer solution ( $\mathrm{pH} 7.4)$ for 2 hours at room temperature. Specimens were dehydrated in ethanol, cleared in propylene oxide, and embedded in an Epon mixture (Poly/Bed 812; Polysciences Inc., USA). Ultrathin sections of Epon-embedded fertilized egg envelope were taken with an Ultracut E (Reichert-Jung, Austria) ultramicrotome at a thickness of about $60 \mathrm{~nm}$. Tissue sections were mounted onto copper grids, double stained with uranyl acetate followed by lead citrate, and observed with a TEM (JEM 1200EX-II; JEOL, Japan) at $80 \mathrm{kV}$. For scanning electron microscope (SEM) observation, prefixation, postfixation and dehydration were conducted by following the same procedure as that for TEM. The samples were replaced with isoamyl acetate and critical point dried. The samples were coated with gold-palladium using an ion sputter (JFC-1100; JEOL). Subsequently, the outer surface of the fertilized egg envelope were observed with a table top SEM (TM-1000; Hitachi, Japan).

\section{RESULTS}

\section{Morphology of Fertilized Eggs}

Fertilized eggs of N. guentheri and N. patrizii were spherical, yellowish, non-transparent, demersal and adhesive, and had a one-sided large oil droplet in vitelline membrane. There were no morphological differences between two species under the light microscope (Fig. 1). But, the sizes of fertilized egg were different. The size of the fertilized eggs of $N$. guentheri was $0.82 \pm 0.02 \mathrm{~mm}(\mathrm{n}=10)$. That of $N$. patrizii was $0.91 \pm 0.02 \mathrm{~mm}$ $(\mathrm{n}=10)$. The diameter size of oil droplet at both of the species was about $0.35 \pm 0.02 \mathrm{~mm}(\mathrm{n}=10)$, and the perivitelline space was almost not developed in both species.

\section{Outer Surfaces of the Fertilized Egg Envelopes}

Micropyle was not on the outer surface of fertilized egg envelope in both species. In N. guentheri, the whip-like structures (adhesive filaments) were distributed throughout egg envelope (Fig. 2A). The adhesive filaments were covered with fibrous structures (Fig. 2B). Also, that of N. patrizii was distributed throughout egg envelope like a that of $N$. guentheri (Fig. 2C). But the outer surface of adhesive filaments was smooth (Fig. 2D). The adhesive filaments of N. guentheri were bigger than that of $N$. patrizii. The size of adhesive filaments of $N$. guentheri was the major axis 35 to $45 \mu \mathrm{m}$, the minor axis 4 to $5 \mu \mathrm{m}$, and that of $N$. patrizii was the major axis 30 to 35 $\mu \mathrm{m}$, the minor axis 1.4 to $1.8 \mu \mathrm{m}$. Also, the outer surface of fertilized egg envelope in N. guentheri was rough (Fig. 2B) and that of N. patrizii was porous (Fig. 2D).

\section{Fertilized Egg Envelope Sections}

In N. guentheri, the thickness of the fertilized egg envelope was about 17 to $19 \mu \mathrm{m}$, and the egg envelope consisted of two distinct layers: an outer, electron-dense layer containing 

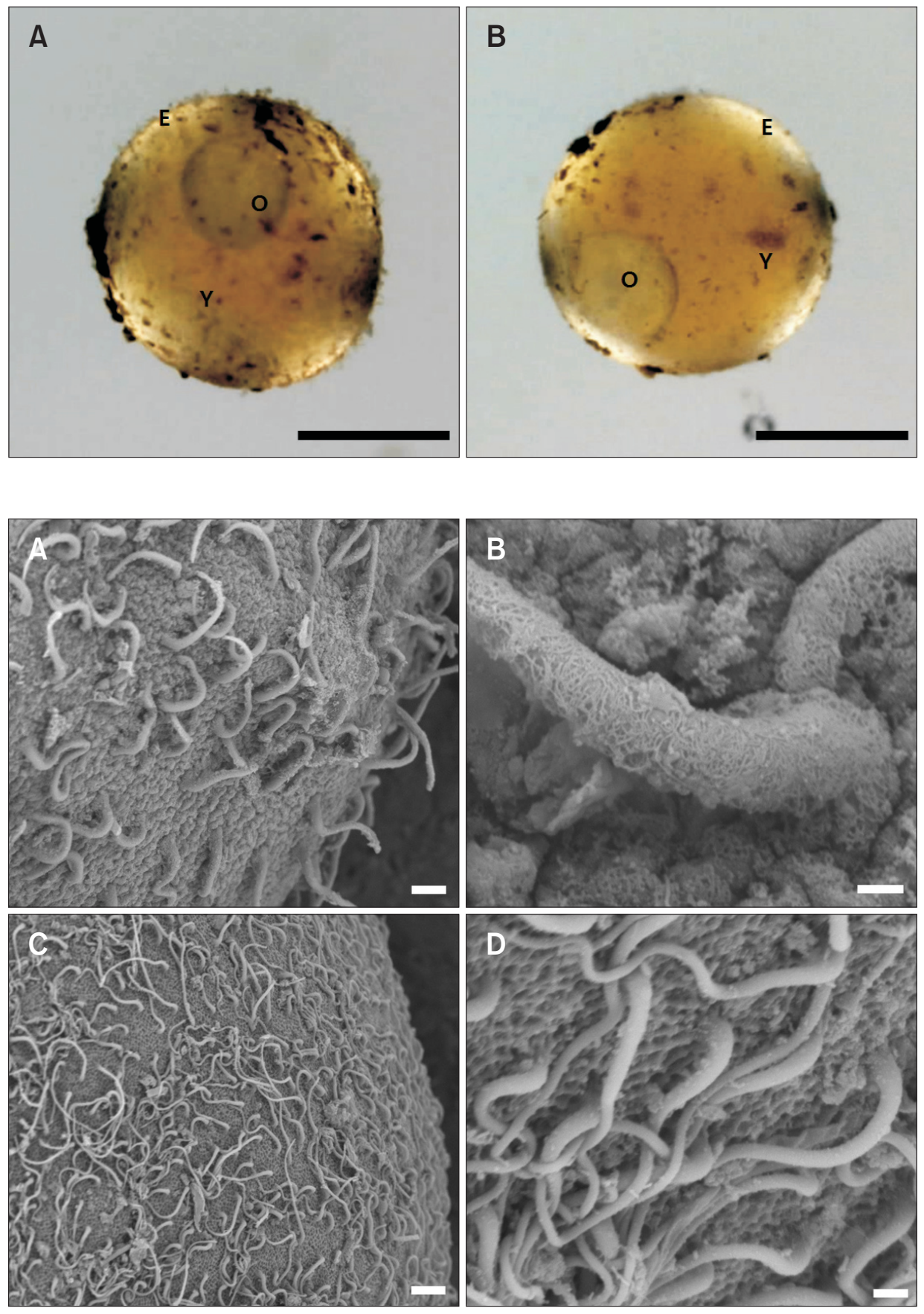

Fig. 1. (A) The fertilized egg of Nothobranchius guentheri. (B) The fertilized egg of Nothobranchius patrizii. There were no morphological differences between two species under the light microscope. Scale bars $=500 \mu \mathrm{m}$. E, egg envelope; O, oil droplet; $\mathrm{Y}$, yolk.
Fig. 2. (A) Scanning electron micrograph of outer surface on the egg envelope of Nothobranchius guentheri. The adhesive filaments were distributed throughout egg envelope (scale bar $=10 \mu \mathrm{m}$ ). (B) Magnified adhesive filaments in $N$. guentheri. The adhesive filaments were covered with fibrous structures (scale bar $=2 \mu \mathrm{m}$ ). (C) Outer surface of the egg envelope in Nothobranchius patrizii. The adhesive filaments were distributed throughout egg envelope (scale bar=10 $\mu \mathrm{m}$ ). (D) Magnified outer surface of the egg envelope in $N$. patrizii. The outer surface of fertilized egg envelope was porous and the outer surface of adhesive filaments were smooth (scale bar $=2 \mu \mathrm{m})$. adhesive filaments and an inner layer of 16 to 17 horizontal electron-dense lamellae alternating with 15 to 16 interlamellae of lower electron density (Fig. 3A). The adhesive filaments was surrounded by electron dense outer layer of egg envelope and filled with electron dense materials (Fig. 3B and C).

In $N$. patrizii, the thickness of the fertilized egg envelope was about 17 to $19 \mu \mathrm{m}$, and the egg envelope consisted of two distinct layers: an outer, electron-dense layer containing adhesive filaments and an inner lamellae layer such as that of N. guentheri (Fig. 4A). In both species, there is no ultrastructural difference in the section of fertilized egg envelope. Adhesive filaments were surrounded by electron dense outer layer of egg envelope and filled with electron dense materials such as N. guentheri (Fig. 4B and C). 


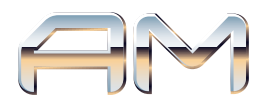

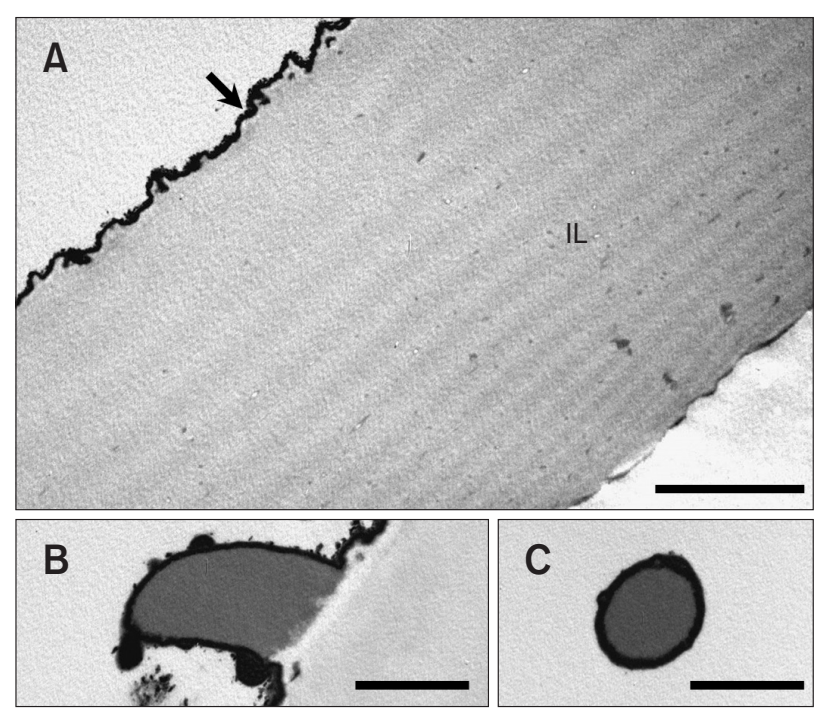

Fig. 3. (A) Transmission electron micrograph of the fertilized egg envelope in Nothobranchius guentheri (scale bar $=5 \mu \mathrm{m}$ ). IL, inner layer; arrow, outer layer. (B) Oblique section of adhesive filament (scale bar=2 $\mu \mathrm{m})$. (C) Cross section of adhesive filament (scale bar $=1 \mu \mathrm{m}$ ).

\section{DISCUSSION}

In teleost, the external shapes of most fertilized eggs are spherical for Cyprinidae (Kim et al., 1998a) and Characidae (Kim et al., 1996), oval for Cichlidae (Kim et al., 2009), and long ellipsoidal for Eleotrididae (Kim et al., 2002) and Pomacentridae (Kim et al., 1998b). In this study, fertilized eggs of N. guentheri and N. patrizii were spherical such as that of Cyprinidae and Characidae and had a large oil droplet in vitelline membrane like fishes belong to Belontiidae. The oil droplet used to make buoyancy of pelagic eggs in that of Belontiidae (Kim et al., 1999), but it seems to used to nutrients for development of embryo in these species. The fertilized eggs had very similar morphology in both species. There were no morphological differences under the light microscope, and the perivitelline space was almost not developed in both species. It seems to do not need to development of perivitelline space because these fishes are egg scatter but the fertilized eggs are of the adhesive type and nearly no physical impact because it spawns on the spawning place such as Cichlidae fishes (Denug et al., 1997). The formation of a perivitelline space is known to follow swelling of the egg envelope due to osmotic pressure caused by the cortical granule reaction (Laale, 1980). These morphological characteristics of fertilized egg such as spherical, yellowish, non-transparent, demersal and adhesive, and a large oil droplet seem to be a common trait for Nothobrachiidae. And we couldn't find micropyle in both species under light microscope as well as SEM because the adhesive filaments were distributed on the outer surface.

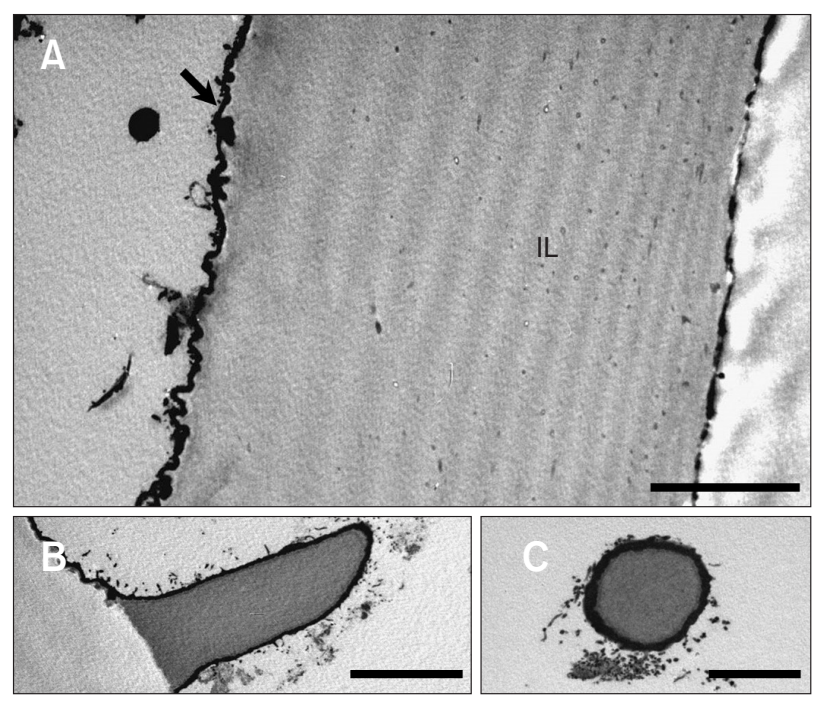

Fig. 4. (A) Transmission electron micrograph of the fertilized egg envelope in Nothobranchius patrizii (scale bar $=5 \mu \mathrm{m}$ ). IL, inner layer; arrow, outer layer. (B) Oblique section of adhesive filament (scale bar=5 $\mu \mathrm{m})$. (C) Cross section of adhesive filament (scale bar $=2 \mu \mathrm{m}$ ).

The adhesive filaments were distributed throughout outer surface of egg envelope in both species. But the ultrastructure of adhesive filaments was differed in size and morphology each other. Also, the outer surface of fertilized egg envelope in N. guentheri was rough and that of N. patrizii was porous. These ultrastructural characteristics can be used to species classification because these traits are species specificity. In Lampetra fluviatilis and Lampetra planeri, two-thirds of the fertilized eggs were covered by adhesive filaments (Kille, 1960). Tomato clown anemonefish (Kim et al., 1998b) and dark sleepers (Kim et al., 2002) have a bundle of adhesive filaments. For fish belong to Cichlidae, reticular structures showed species specificity (Deung et al., 1997). According to the comparative ultrastructure of fertilized egg and fertilized egg envelope in Danio rerio and D. rerio var. frankei, although morphology of fertilized eggs were same, the numbers of appendicular knob-like structures and semihemispherelike structures per unit area on the outer surface displayed definite species specificity. It is known to the ultrastructure of fertilized egg envelope in the zebrafish could be differentiated by mutation (Meyer et al., 1993).

In fine structure of section of fertilized egg envelope, that of both species consisted of two distinct layers: an outer, electron-dense layer containing adhesive filaments and an inner lamellae of lower electron density, and the adhesive filaments were surrounded by electron dense outer layer of egg envelope and was filled with electron dense materials. These ultrastructural characteristics showed similar pattern, and same thickness.

The section of ultrastructure of the fertilized egg envelope 
showed species specificity, even within a same family such as Hemigrammus ocellifer, Gymnocorymbus ternetzi and Hemigrammus caudovittatus belong to Characidae (Kim et al., 1996), Cichlasoma severum, Cichlasoma nigrofasciatum, Cichlasoma managuensis and Symphysodon aequifasciatus belong to Cichlidae (Deung et al., 1997; Kim et al., 2009), D. rerio, Barbus titteya and Tanichthys albonubes belong to Cyprinidae (Kim et al., 1998a) and pink salmon, chum salmon and chinook salmon belong to Salmonidae (Schmehl \& Graham, 1987). However, the fertilized egg envelopes of three species, Trichogaster trichopterus, Trichogaster leeri and Trichogaster trichopterus trichopterus belong to Belontiidae have been reported to have the same structure (Kim et al., 1999).

The external shapes of the fertilized eggs of $N$. guentheri and $N$. patrizii could not be distinguished with the naked eye or under the light microscope. By contrast, the ultrastructure on the adhesive filaments, outer surface except section of fertilized egg envelope displayed definite species specificity. Collectively, our data indicate that these ultrastructural characteristics of adhesive filament and outer surface can be used for classification of species.

\section{CONCLUSIONS}

We compared the fertilized egg morphology and ultrastructures of surface structures and the cross section of fertilized egg envelopes of $N$. guentheri and N. patrizii using a light and electron microscopes. The external shapes of fertilized egg and section of fertilized egg envelope were same, but ultrastructure of adhesive filaments on the outer surface was concluded to show species specificity. Our data indicate that the ultrastructural differences of adhesive filament of fertilized egg envelope and outer surface show species specificity although these species belong to same genus.

\section{CONFLICT OF INTEREST}

No potential conflict of interest relevant to this article was reported.

\section{REFERENCES}

Bailey M and Sandford G (1998) The New Guide to Aquarium Fish, a Comprehensive and Authoritative Guide to Tropical Freshwater, Brackish, and Marine Fishes (Hermes House, London).

Brummett A R and Dumont J N (1981) A comparison of chorions from eggs of northern and southern populations of Fundulus heteroclitus. Copeia 3, 607-614.

Cameron I L and Hunter K E (1984) Regulation of the permeability of the medaka fish embryo chorion by exogeneous sodium and calcium ions. J. Exp. Zool. 231, 447-454.

Deung Y K, Kim D H, and Reu D S (1999) Ultrastructure of gametes in the three-spine stickleback, Gasterosteus aculeatus aculeatus. Korean J. Microsc. 29, 177-187.

Deung Y K, Reu D S, and Kim D H (1997) Comparative ultrastructures of the fertilized egg envelopes in golden severum, convic cichlid and discus, Cichlidae, teleost. Korean J. Microsc. 27, 417-432.

Donovan M J and Hart N H (1986) Cortical granule exocytosis is coupled with membrane retrieval in the egg of Brachydanio. J. Exp. Zool. 237, 391-405.

Harvey B, Kelley R N, and Ashwood-Smith M J (1983) Permeability of intact and dechorionated zebra fish embryos to glycerol and dimethyl sulfoxide. Cryobiol. 20, 432-439.

Huber J H (1996) Killi-Data 1996: Updated Checklist of Taxonomic Names, Collecting Localities and Bibliographic References of Oviparous Cyprinodont Fishes (Atherinomorpha, Pisces) (Société Française d'Ichtyologie, Muséum National d'Histoire Naturelle, Paris).

Ivankov V N and Kurdyayeva V P (1973) Systematic differences and the ecological importance of the membranes in fish eggs. J. Ichthyol. 13, 864-873.

Kawaguchi M, Yasumasu S, Shimizu A, Sano K, luchi I, and Nishida M (2010) Conservation of the egg envelope digestion mechanism of hatching enzyme in euteleostean fishes. FEBS J. 277, 4973-4987.

Kille R A (1960) Fertilization of the lamprey egg. Exp. Cell Res. 20, $12-27$.

Kim D H, Chang B S, Teng Y C, Kim S, Joo K B, and Lee K J (2009) Ultrastructure of the fertilized egg envelope in Cichlasoma managuensis, Cichlidae, Teleost. Korean J. Microsc. 39, 9-15.

Kim D H, Deung Y K, Kim W J, Reu D S, and Kang S J (1999) Comparative ultrastructures of the fertilized egg envelopes from three-spot gourami, pearl gourami and marble gourami, Belontiidae, teleost. Korean J. Microsc. 29, 343-351.

Kim D H, Reu D S, and Deung Y K (1996) A comparative study on the ultrastructures of the egg envelope in fertilized eggs of fishes, Characidae, three species. Korean J. Microsc. 26, 277-291.

Kim D H, Reu D S, and Deung Y K (1998a) Comparative ultrastructures of the fertilized egg envelopes in three species, Cyprinidae, teleost. Korean J. Microsc. 28, 237-253.

Kim D H, Reu D S, and Deung Y K (1998b) Ultrastructure of the fertilized egg envelope in tomato clown anemonefish (Amphiprion freatus), Pomacentridae, marine teleost. Korean J. Microsc. 28, 273-282.

Kim D H, Reu D S, and Deung Y K (2002) Ultrastructure of the fertilized egg envelope from dark sleeper, Eleotrididae, teleost. Korean J. Microsc. 32, 39-44.

Laale H W (1980) The perivitelline space and egg envelopes of bony fishes: a review. Copeia 2, 210-226.

Lönning S (1972) Comparative electron microscopic studies of teleostean eggs. Sarsia 49, 41-48.

Meyer A, Biemann C H, and Orti G (1993) The phylogenetic position of the zebrafish (Danio rerio), a model system in developmental biology: an invitation to the comparative method. Proc. Biol. Sci. 252, 231236. 
Schmehl M K and Graham E F (1987) Comparative ultrastructure of the zona radiata from eggs of six species of salmonids. Cell Tissue Res. 250, 513-519.
Stehr C M and Hawkes J W (1979) The comparative ultrastructure of the egg membrane and associated pore structures in the starry flounder, Platichthys stellatus (Pallas), and pink salmon, Oncorhynchus gorbuscha (Walbaum). Cell Tissue Res. 202, 347-356. 